\title{
Accelerated Neutrophil Apoptosis in 2 Canine Cases of Hepatic Disorder
}

\author{
Keisuke OGUMA $^{1)}$, Jun-ichi SANO ${ }^{1)}$, Rui $\mathrm{KANO}^{1) *}$, Toshihiro WATARI ${ }^{2)}$ and Atsuhiko HASEGAWA ${ }^{1)}$ \\ ${ }^{1)}$ Departments of Pathobiology and ${ }^{2)}$ Comprehensive Veterinary Clinical Studies, Nihon University School of Veterinary Medicine, 1866 \\ Kameino, Fujisawa, Kanagawa 252-8510, Japan
}

(Received 13 December 2001/Accepted 11 December 2002)

ABSTRACT. Accelerated neutrophil apoptosis was confirmed by TUNEL assay in two canine cases of hepatic disorder. One dog was diagnosed as having lymphocytic hepatitis and the other lymphocytic cholangitis by histopathology of liver biopsy specimen. KEY WORDS: apoptosis, inflammatory hepatic disorder, neutrophil.

The neutrophil (PMN: polymorphonuclear leukocyte) plays a major role in biophylaxis against many infectious agents. PMNs have the shortest life span of all leukocytes, surviving in peripheral blood for hours to days before being apoptotic. Neutropenia is frequently detected in human cirrhotic patients, predisposing to bacterial infections, and it appears to be related to the increased PMN apoptosis [5]. However, the relations between PMN apoptosis and diseases have not been well investigated, and few discussions have been reported on PMN apoptosis in dogs. Previously, rates of PMN apoptosis in healthy dogs have been determined by the TUNEL assay [11], however those in diseased dogs have not been reported. In the present study, the accelerated apoptosis of PMNs was confirmed in two patient dogs diagnosed as having inflammatory hepatic disease (Cases 1 and 2).

Case 1 was a four-year and six-month-old, spayed female Labrador retriever and referred to us with chronic vomiting and diarrhea. In blood analyses of plasma enzymes associated with liver disorders, total bile acid and total bilirubin levels were conspicuously high. Lymphocyte count was decreased and eosinophils were not detected in complete blood count (CBC) (Table 1). Prothrombin time (PT) and activated partial thromboplastin time (APTT) were 10.2 and 14 seconds, respectively. The histopathological diagnosis of a liver biopsy specimen was lymphocytic hepatitis. The dog was treated with prednisolone, ursodeoxycolic acid, amoxicillin, metronidazole, vitamin $\mathrm{K}$ and amino acid solution. The dog's condition gradually improved and it was discharged from hospital. PMN isolation was carried out on the second day of hospitalization.

Case 2 was a female, two-year and eight-month-old Borzoi and admitted with anorexia and jaundice. Total bile acid and total bilirubin levels were conspicuously high in blood chemistry. Lymphocytes were decreased in number and eosinophils were not detected (Table 1). PT and APTT were 8.2 and 12 seconds, respectively. Lymphocytic cholangitis was diagnosed by histopathological examination of a liver biopsy specimen. The dog was initially treated with ursode-

\footnotetext{
* Correspondence to: Kano, R., Department of Pathobiology, Nihon University School of Veterinary Medicine, 1866 Kameino, Fujisawa, Kanagawa 252-8510, Japan.
}

oxycolic acid, ampicillin, glutathione and glycyrrhizin, and late additional medications included prednisolone, azathioprine, metronidazole, misoprostol and carbazochrome. Despite treatment, complications with disseminated intravascular coagulation appeared and the dog died. PMN isolation was achieved on the third day of hospitalization.

The procedure of PMN isolation was reported previously [11]. PMNs separated from peripheral blood were resuspended in RPMI 1640 medium supplemented with $10 \%$ fetal calf serum. Final concentration of the cells was $4 \times 10^{6}$ cells $/ \mathrm{m} l$. These cell suspensions were incubated at $3 \mathrm{P}^{\circ} \mathrm{C}$ for various time periods. Terminal deoxynucleotidyl transferase-mediated deoxyuridine triphosphate nick end labeling (TUNEL) assay was performed using DeadEnd Colorimetric Apoptosis Detection System (Promega, Madison, U.S.A.). After incubation for $0,4,8,12$ and $24 \mathrm{hr}$, PMNs from patient and control dogs were stained according to the manufacturer's instructions. At least 500 cells were evaluated in each preparation. The TUNEL-positive and negative cells were counted and apoptotic cell rates were calculated.

The apoptotic PMN rates of 2 patient dogs and 3 healthy control dogs (A, B, C) are given in Table 2 and Fig. 1.

Apoptotic PMNs of both patient dogs were markedly higher than those of the healthy dogs. The result was consistent with the results of human cirrhotic patients, and the correlation between PMN apoptosis and diseases should be further investigated in a large number of cases. Not only in dog but also in human, the concrete mechanisms of accelerated PMN apoptosis in liver disorders remain unknown.

Many factors have been reported to affect PMN apoptosis [1]. For instance, tumor necrosis factor (TNF)- $\alpha$ and Fas ligand could promote PMN apoptosis, while interleukin (IL)-1 $\beta$, IL-2, IL-8, granulocyte-macrophage colony stimulating factor, glucocorticoids and leukotriene B4 would suppress it $[4,6,7,13]$. It is interesting that only a few factors have been reported as pro-apoptotic extracellular factors for PMN, while many factors have been reported as anti-apoptotic factors. It was shown that TNF- $\alpha$ and IL- 8 levels were elevated in cases of child autoimmune hepatitis [10]. Some reports also note that nitric oxide (NO) was produced from neutrophils or monocytes in human liver cirrhosis or dys- 
Table 1. CBC and blood chemistry of dogs with hepatitis and control dogs

\begin{tabular}{|c|c|c|c|c|c|c|c|}
\hline & unit & Case 1 & Case 2 & \multicolumn{2}{|c|}{ Control A } & Control B & Control C \\
\hline $\mathrm{RBC}$ & $\times 10^{6} / \mu l$ & 6.71 & 5.58 & \multicolumn{2}{|c|}{8.36} & 6.59 & 8.19 \\
\hline $\mathrm{Hb}$ & $\mathrm{g} / \mathrm{d} l$ & 16.1 & 13.7 & \multicolumn{2}{|c|}{18.8} & 14.6 & 17.4 \\
\hline Hct & $\%$ & 50.3 & 45.1 & \multicolumn{2}{|c|}{58.3} & 46.0 & 53.4 \\
\hline $\mathrm{MCV}$ & $\mathrm{f} l$ & 75 & 81 & \multicolumn{2}{|c|}{70} & 70 & 65 \\
\hline MCHC & $\mathrm{g} / \mathrm{d} l$ & 32.0 & 30.4 & \multicolumn{2}{|c|}{32.2} & 31.7 & 32.6 \\
\hline WBC & $/ \mu l$ & 9900 & 9600 & \multicolumn{2}{|c|}{10600} & 10200 & 9300 \\
\hline band & $\mid \mu l$ & 99 & 288 & \multicolumn{2}{|c|}{106} & 51 & 186 \\
\hline seg & $/ \mu l$ & 8811 & 8160 & \multicolumn{2}{|c|}{7208} & 6987 & 5022 \\
\hline lym & $/ \mu l$ & 792 & 768 & \multicolumn{2}{|c|}{2544} & 2132 & 3441 \\
\hline mono & $/ \mu l$ & 198 & 384 & \multicolumn{2}{|c|}{530} & 714 & 279 \\
\hline eosino & $\mid \mu l$ & 0 & 0 & \multicolumn{2}{|c|}{212} & 316 & 372 \\
\hline baso & $/ \mu l$ & 0 & 0 & \multicolumn{2}{|c|}{0} & 0 & 0 \\
\hline PLT & $\times 10^{3} / \mu l$ & 281 & 335 & \multicolumn{2}{|c|}{239} & 344 & 215 \\
\hline ALP & $\mathrm{U} / l$ & 303 & 2449 & \multicolumn{2}{|c|}{120} & 95 & 91 \\
\hline ALT & $\mathrm{U} / l$ & 681 & 758 & \multicolumn{2}{|c|}{67} & 81 & 54 \\
\hline AST & $\mathrm{U} / l$ & 119 & 191 & \multicolumn{2}{|c|}{32} & 25 & 21 \\
\hline GGT & $\mathrm{U} / l$ & 52 & 51 & \multicolumn{2}{|c|}{4} & 0 & 3 \\
\hline T-Bil & $\mathrm{mg} / \mathrm{d} l$ & 16.67 & 12.44 & \multicolumn{2}{|c|}{5.47} & 7.18 & 3.76 \\
\hline T-BA & $\mu \mathrm{mol} / l$ & 27 & 285 & \multicolumn{2}{|c|}{-} & - & - \\
\hline ALB & $\mathrm{g} / \mathrm{d} l$ & 2.83 & 2.70 & \multicolumn{2}{|c|}{3.23} & 3.56 & 2.91 \\
\hline $\mathrm{TP}$ & $\mathrm{g} / \mathrm{d} l$ & 6.8 & 7.2 & \multicolumn{2}{|c|}{6.8} & 7.0 & 6.4 \\
\hline $\mathrm{RBC}$ & \multicolumn{3}{|l|}{ : red blood cell } & \multicolumn{2}{|r|}{ ALB } & \multicolumn{2}{|l|}{ : albumin } \\
\hline $\mathrm{Hb}$ & \multicolumn{3}{|l|}{ : hemoglobin } & \multicolumn{2}{|r|}{ ALP } & \multicolumn{2}{|c|}{ : alkaline phosphatase } \\
\hline Hct & : hematocrit & & & & ALT & : alanine a & inotransferase \\
\hline MCV & : mean corpuscula & olume & & & AST & : aspartate & minotransferase \\
\hline $\mathrm{MCHC}$ : & : mean corpuscula & emoglobi & concent & ation & GGT & $: \gamma$-glutam & 1 transpeptidase \\
\hline WBC & : white blood cell & & & & T-Bil & : total bilir & bin \\
\hline band & : band nuclear neu & phil & & & T-BA & : total bile & acid \\
\hline seg & : segmented nucle & neutrophi & & & TP & : total prot & \\
\hline $1 \mathrm{ym}$ & lymphocyte & & & & & & \\
\hline mono & monocyte & & & & - & : not deter & hined \\
\hline eosino & : eosinophil & & & & & & \\
\hline baso & : basophil & & & & & & \\
\hline PLT & : platelet & & & & & & \\
\hline
\end{tabular}

Table 2. Apoptotic PMN rate (\%) in each dog

\begin{tabular}{lrrccc}
\hline & Case 1 & Case 2 & Control A & Control B & Control C \\
\hline $0 \mathrm{hr}$ & 3.1 & 0.0 & 0.0 & 0.0 & 0.5 \\
$4 \mathrm{hr}$ & 6.3 & 8.2 & 0.0 & 0.8 & 0.4 \\
$8 \mathrm{hr}$ & 20.5 & 42.7 & 0.5 & 2.0 & 0.4 \\
$12 \mathrm{hr}$ & 51.2 & 71.7 & 5.4 & 17.8 & 3.2 \\
$24 \mathrm{hr}$ & 68.8 & 88.0 & 25.9 & 35.2 & 20.9 \\
\hline
\end{tabular}

function, and that it would accelerate PMN apoptosis $[3,8$, 12]. However, cytokine and NO levels have not been evaluated in dogs with hepatic disorders. The level of plasma diverse cytokines and other mediators would be high in canine cases of inflammatory hepatic disorders, and they might induce PMN apoptosis.

Many genes, such as $b c l-2$ family, were also reported as regulating PMN apoptosis, but they are only a few reports of the relation between cytokines and bcl-2 family genes [2,9].

Henceforth, further investigations are required into PMN apoptosis in the diseased condition and they should include cytokines, other mediators and molecular analyses. Measurement of the apoptotic rate of PMNs might be indicative

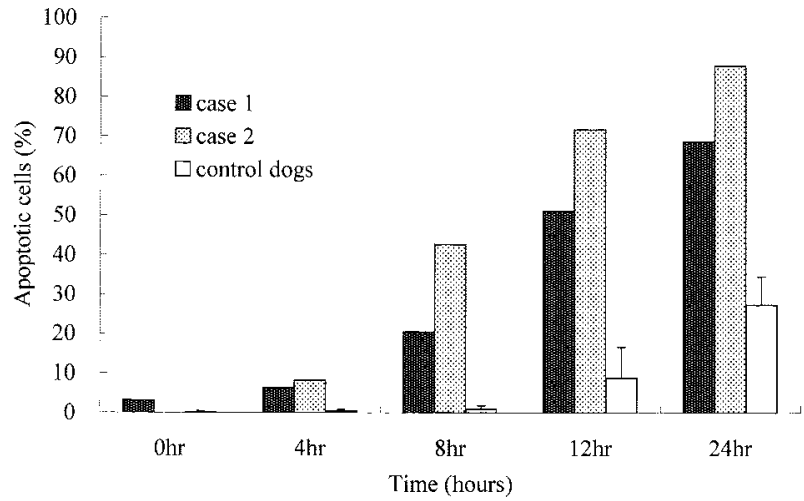

Fig. 1. PMN apoptotic rate in controls and patient dogs. Vertical bars represent mean \pm standard deviation of control dogs.

of the pathophysiological state of liver disorders.

\section{REFERENCES}

1. Akgul, C., Moulding, D.A. and Edwards, S.W. 2001. FEBS Letters 487: 318-322. 
2. Dibbert, B., Weber, M., Nikolaizik, W.H., Vogt, P., Schoni, M.H., Blaser, K. and Simon, H.U. 1999. Proc. Natl. Acad. Sci. U.S.A. 96: 13330-13335.

3. Galley, H.F., Coomansingh, D., Webster, N.R. and Brunt, P.W. 1998. Clin. Sci. 95: 355-359.

4. Klein, J.B., Buridi, A., Coxon, P.Y., Rane, M.J., Manning, T., Kettritz, R. and McLeish, K.R. 2001. Cell. Signal. 13: 335343.

5. Kusaba, N., Kumashiro, R., Ogata, H., Sata, M. and Tanikawa., K. 1998. Intern. Med. 37: 11-17.

6. Liles, W.C., Kiener, P.A., Ledbetter, J.A., Aruffo, A. and Klebanoff, S.J. 1996. J. Exp. Med. 184: 429-440.

7. Liles, W.C. and Klebanoff, S.J. 1995. J. Immunol. 155: 32893291.
8. Masini, E., Mugnai, L., Foschi, M., Laffi, G., Gentilini, P. and Mannaioni, P.F. 1995. Int. Arch. Allergy Immunol. 107: 197198.

9. Moulding, D.A., Quayle, J.A, Hart, C.A. and Edwards, S.W. 1998. Blood 92: 2495-2502.

10. Neuman, M.G. 2001. Crit. Rev. Clin. Lab. Sci. 38: 109-166.

11. Oguma, K., Kano, R. and Hasegawa, A. 2000. Vet. Immunol. Immunopathol. 76: 157-162.

12. Ward, C., Wong, T.H., Murray, J., Rahman, I., Haslett, C., Chilvers, E.R. and Rossi, A.G. 2000. Biochem. Pharmacol. 59: 305-314.

13. Webb, P.R., Wang, K.Q., Scheel-Toellner, D., Prongracz, M., Salmon, M. and Lord, J.M. 2000. Apoptosis 5: 451-458. 